\title{
Optimizing Quality of Care for Septic Patients in Developing Countries
}

\author{
Jasmine Saleh* \\ National Institute of Health, Bethesda, USA
}

Received: December 28, 2014; Accepted: February 16, 2015, Published: March 10, 2015

*Corresponding author: Jasmine Saleh, National Institute of Health, MD, USA Pin: 20892; Tel: +217-303-5538; E-mail: jasminesaleh@gmail.com

\begin{abstract}
Sepsis is a major cause of morbidity and mortality in intensive care units. It affects millions of people worldwide annually, causing hundreds of thousands deaths and health-care expenditure of at least $\$ 16.7$ billion per year. This highlights the significance of diagnosing and treating septic patients emergently. Unfortunately, developing countries are struggling with limited resources, lack of educational efforts targeting sepsis, and lack of access to healthcare, hence making it difficult for the healthcare professionals to understand the disease process as well as to recognize and manage sepsis. This review paper will discuss possible strategies for the developing countries to decrease their disproportionately high incidence and mortality of sepsis.
\end{abstract}

Keywords: Sepsis; Septic shock; Developing countries

\section{Introduction}

Sepsis is a major cause of morbidity and mortality in noncoronary intensive care units. It affects millions of people around the world annually and accounts for $60-80 \%$ of deaths in the developing countries [1]. The incidence is increasing at the rate of approximately $8.76 \%$ each year, regardless of technical developments in intensive care units and advanced supportive treatment [2]. Sepsis has been a tremendous burden to the health care systems worldwide, with costs estimated to be at least $\$ 17.0$ billion per year [3]. This highlights not only the significance of sepsis' contribution to morbidity and economic costs but also the clinical need to diagnose and treat sepsis urgently, improve our understanding of sepsis, and develop novel treatment strategies for septic patients. Unfortunately, developing countries are struggling with limited resources, absence of educational efforts targeting sepsis, and lack of access to healthcare, which make it difficult for the healthcare providers to understand the disease process as well as to recognize and manage sepsis [4-9]. The purpose of this article is to recommend several strategies to improve clinical outcomes in patients with sepsis in developing countries.

\section{Discussion}

\section{Burden of sepsis in developing world}

Sepsis is a major source of morbidity and mortality in developing countries. Although data on the burden of sepsis in developing countries is lacking in the published literature, the incidence and mortality are likely to be disproportionally high in developing countries compared to developed countries [4-10]. The Brazilian Sepsis Epidemiological Study has confirmed this conclusion by evaluating 1383 patients who were admitted to five different intensive care units. The investigators discovered that the incidence of sepsis, severe sepsis, and septic shock were 61.4, 35.6, and 30.0 per 1000 days, respectively [6]. The mortality of patients with sepsis, severe sepsis, and septic shock were $34.7 \%, 47.3 \%$, and $52.2 \%$, respectively [6]. These high incidences and mortalities of sepsis in developing countries may be caused by their quality of healthcare, lack of access to healthcare and educational efforts targeting sepsis, and shortage of resources.

It is also important to note that severe sepsis is likely to complicate malnutrition, malaria, maternal conditions, and chronic illnesses, including HIV/AIDS, diabetes, and cancer in developing countries [4-5,8]. For instance, Bane et al.[7] Evaluated HIV-positive patients who were admitted to an Ethiopian hospital and noticed that sepsis and septic shock were among the most common causes of mortality due to their immunosuppressant $[4,7]$. Sani et al. $[4,8]$. Reviewed the causes of death among patients with AIDS over 4 years in a Nigerian hospital As a result of their calculations, 455 of 4574 patients (9.9\%) were admitted due to their HIV/AIDS-related diagnoses, which caused 176 deaths $(38.7 \%)$ over the period[4,8]. The most common causes of these deaths were tuberculosis (33.4\%) and sepsis (23.8\%). This indicates that immunosuppressant secondary to acute illnesses and chronic conditions in developing countries may thus potentially significantly increase the incidence and mortality of sepsis in developing countries [4].similarly, a large prospective study evaluating 1,235 Brazilian patients admitted to 90 Brazilian icus demonstrated that there was a clear association between infection and mortality [10]. Furthermore, the overall ICU mortality rate was $28.4 \%$ with the higher rate in the infected patients than in non-infected patients (37.6\% vs $13.2 \%$ ) [10]. Likewise, the in-hospital mortality rate was $34.2 \%$, with $44.2 \%$ in the infected patients and $17.7 \%$ in non-infected patients. The authors performed a multivariate analysis and concluded that the significant factors associated with the incidence of infection were emergency surgery (OR 2.89), mechanical ventilation (OR 
2.06), and the SAPS II score (OR 1.04) [10]. The main factors related to mortality were ICC functional class III/ IV (OR 3.0), diabetes mellitus (OR 0.48), cirrhosis (OR 4.62), male gender (OR 0.68), mechanical ventilation (OR 1.87), hemodialysis (OR 1.98), and the SAPS II score (OR 1.08) [10].This is crucial for the physicians and healthcare providers to be aware, since there is a high prevalence of comorbidities and chronic medical conditions in developing countries.

\section{Optimizing management of sepsis in developing countries}

Improve access to healthcare: Developing countries lack simple access to health care and thus implementation of clinical protocols and guidelines to manage sepsis [9]. This leads to the delays in care and lack of recognition or misdiagnosis of sepsis, hence resulting in increased morbidity and mortality. A multicenter observational study evaluated the factors associated with mortality in 396 septic patients admitted to ICUs in 19 public and private institutions in Brazil in 2013 [11]. The authors observed that private hospitals had a higher chance of diagnosing sepsis within two hours of the onset of organ dysfunction in addition to having more compliance implementing the treatment protocol [11]. Delayed recognition of sepsis and inadequate treatment of sepsis due to lack of access to emergency departments and intensive-care units, shortage of resources, and limited beds in public hospitals may contribute to its higher sepsis mortality rate. Becker et al. $[9,11]$ proposed a possible solution to this issue by recommending that developing countries should establish a systematic approach to create an efficient triage in order to improve access to emergency care and intensive care services[4]. This would enable the healthcare providers to identify critically ill patients and provide them with basic interventions without any delay to reaching definitive care. Becker et al. [4] suggests that an efficient triage would therefore "underpin the apportionment of resources and attention to those in greatest acute need, an issue of integral importance in resource-constrained settings."

Improve diagnostic laboratory testing: Proper diagnostic testing is crucial for the healthcare professionals to effectively manage septic patients. Unfortunately, resources and technologies are limited and not widely available in developing countries [4]. Cheng et al. [5] proposes that the possible solutions for this significant issue would be the development of low-cost laboratories and simple, cost-effective standardized laboratory testing. For instance, there is rapid, inexpensive testing available for lactate, which is a marker for poor tissue perfusion and thus increased in septic patients [4].Therefore, it may be valuable for the physicians to monitor lactate concentrations in patients with sepsis and use this critical information to determine diagnosis and response to treatment [4].

Execute an early, cost-effective intervention: Healthcare providers in developing countries need to understand that prompt recognition and intervention are essential and costeffective strategies to manage sepsis [5,9]. Li et al. [12] investigated the compliance with the Surviving Sepsis Campaign protocols and resultant mortality in septic patients hospitalized in ICUs in China. The overall compliance during 6-hour resuscitation and 24-hour management bundles were $5.5 \%$ and $17.4 \%$, respectively. Although the compliance with resuscitation was poor, the investigators discovered that 6-hour resuscitation bundle was significantly associated with decreased 28-day mortality [12].

Unfortunately, the developing countries lack the resources to perform even minor interventions, such as administrating intravenous fluid, and to monitor the patients' response to treatment [4]. Nevertheless, basic sepsis inventions can still be achieved with simple fluid resuscitation and management of blood pressure [5]. In developed countries, crystalloid fluids remain the most commonly used for intravenous fluid administration in order to restore organ perfusion.[10,13] Recent data has concluded that the use of oral rehydration salts to treat dehydration from diarrhea is well established.[4] Since sepsis and dehydration from diarrhea both cause intravascular volume depletion and decreased organ perfusion, Becker et al. [4] suggests that oral rehydration salts may be applicable to initial fluid resuscitation in sepsis. As discussed earlier in this article, nor epinephrine and dopamine are used for persistent hypotension following fluid administration $[5,13]$. This may be problematic and not feasible in some developing countries due to their limited resources [5]. However, a study was conducted on oral midodrine, an non-intravenous vasopressor agent, and demonstrated that it increases the blood pressure and decreases the heart rate $[5,14]$. Therefore, it is possible that it can substitute crystalloid in septic management. However, since this study was published in 1979, more studies on oral midodrine and other possible non-intravenous vasopressor agents in sepsis management needs to be performed [5,14]. In addition to performing early interventions in septic patients, it is crucial for the healthcare providers to assess the patients' clinical condition in conjunction with their lactate concentrations to determine the severity of their infection and their responses to treatment [4]. While developed countries may use electronic monitors for hemodynamic monitoring, Cheng et al. [5] recommends that it may be more feasible and cost-effective for developing regions to have the healthcare providers directly observing the patients' clinical status such as blood pressure, heart rate, skin color, capillary refill, mental status, and urinary output. Although it is possible to perform basic sepsis interventions, further research is still required to develop optimal, cost-effective management algorithms for resource-challenged developing countries $[4,5]$.

Utilize early and appropriate antibiotic coverage: Studies have shown that early intervention and administration of appropriate antimicrobial antibiotics are the most efficient and cost-effective strategies to improve outcomes [4-5,12]. Li et al. [12] Demonstrated that compliance with the blood culture before antibiotics protocol produced the most significant decrease in 28-day mortality (OR, 0.33). Therefore, morbidity and mortality would significantly increase if antimicrobials are delayed or are selected without consideration to the source of septic infections $[4,12]$. 
It is important to note that the bacteriology of sepsis across developing countries varies and depends on their regional and population characteristics [4]. For instance, a meta-analysis evaluating 22 studies on 2051 patients with community-acquired sepsis in Africa in 2010 reported that the most common organisms were Salmonella enterica (58.4\%), Mycobacterium tuberculosis (30.7\%), Streptococcus pneumonia (18.3\%), Staphylococcus aureus (9.5\%), and Escherichia coli (7.3)[15]. Therefore, Becker et al. [4] Suggests that appropriate antibiotic treatment for sepsis should also depend on the ecological pressures resulting from local antibiotic availability, patterns of antibiotic use, and antibiotic resistance patterns. Since the spectrum of infectious disease is much broader in developing countries than in most developed countries, Becker et al. [4] Recommends that parasitic, bacterial, and viral causes must be considered. For instance, due to the high prevalence of HIV in many developing countries, fungal and mycobacterial infection should also be considered. This highlights that, the recommended guidelines by Surviving Sepsis Campaign on initial medication choices may be difficult to apply in developing countries [4-5]. It is therefore critical for future research to address these issues.

Create training programs for healthcare professionals: While prompt recognition and management of sepsis are likely to be the most cost-effective interventions for the patients with sepsis in developing countries with limited resources, educational efforts targeted at sepsis in developing countries are necessary and crucial to improve clinical outcomes $[4-5,16]$. The diverse signs and symptoms of sepsis frequently disguised the disease process, and lack of targeted sepsis education in developing countries makes it difficult for the healthcare professionals to make accurate diagnoses and provide care for the patients [4]. Developing countries therefore need educational programs to train healthcare providers on the prevention, early recognition and diagnosis, and management of sepsis. [4-5,16]. This would lead to a higher compliance with the early goal-directed therapy and the Surviving Sepsis Campaign guidelines, which has been demonstrated in a recent interventional study where a network of ten private hospitals in Brazil used a multifaceted, centrally coordinated quality improvement program involving multiple multidisciplinary educational sessions, case managements, and continuous performance assessment [16]. As a result, the healthcare professionals had high compliance with the resuscitation protocol, increasing the proportion of patients who received adequate treatment from $13 \%$ to $62 \%$ and hence decreasing the overall mortality from $55 \%$ to $26 \%$ [16]. This indicates that educational efforts would lead to significant improvements in clinical outcomes in sepsis.

Modify surviving sepsis campaign guidelines: The Surviving Sepsis Campaign guidelines have been developed in response to high morbidity and mortality and also the necessity to improve the management of sepsis [4]. Unfortunately, these issues still persist in developing countries. A possible reason is that the guidelines have failed to incorporate the realities and inequities of different health-care systems and limitation of resources in developing countries. Baelani et al. $[5,9,17]$.
Conducted the survey in African countries and high-income countries to compare their availability of the resources necessary to implement the Surviving Sepsis Campaign guidelines [17]. African hospitals were less likely to have an emergency room ( $85.5 \%$ vs $97.7 \%$ ) or intensive care unit (73.8\% vs. $100 \%)$ than those in high-income countries [17]. Drugs and equipment necessary to implement the Surviving Sepsis Campaign guidelines were also less available in African countries than highincome countries. Unfortunately, of all African countries, only $1.5 \%$ of respondents had the resources available to implement the Surviving Sepsis Campaign guidelines in entirety[17]. This strongly suggests that it may not be possible for Africa to implement the most recent Surviving Sepsis guidelines due to its shortage of required resources [17].Therefore, Surviving Sepsis Campaign needs to modify its guidelines and treatment algorithms to apply in developing countries due to their limited resources and technologies $[5,9,17]$.

Perform more research focusing on Developing World: Most of the studies on sepsis are undertaken in the developed countries and therefore may not be applicable to the developing countries [5]. Further research must be conducted to determine low-cost, relevant diagnostic tests and antimicrobial treatment plans tailored to the developing countries' ecological characteristics and resources [4].Further research is also needed to define and develop the most cost-effective interventions and guidelines targeted at sepsis in developing countries [4].

\section{Conclusion}

The Surviving Sepsis Campaign has created the guidelines of managing sepsis and septic shock. These guidelines focus on adequate fluid resuscitation to restore intravascular volume, vasopressors to maintain MAP, and inotropes to improve myocardial contractility and ensure adequate oxygen delivery. Although sepsis management in developed countries relies on these clinical protocols and guidelines as well as resources that are not widely available in developing countries, prompt recognition and management of sepsis in developing countries are still essential to improve the outcomes of this potentially fatal infection. However, simple, low-cost management algorithms tailored to developing countries' ecology and resources still require further research and development.

\section{Conflicting Interests}

The author declares no conflicts of interest with respect to the authorship and/or publication of this article.

\section{References}

1. Reinhard K, Daniels R, Machado FR. The burden of sepsis: a call to action in support of World Sepsis Day 2013. Rev Bras Ter Intensiva 2013 Jan-Mar; 25(1): 3-5.

2. Martin GS. Sepsis, severe sepsis and septic shock: changes in incidence, pathogens, and outcomes. Expert Rev Anti Infect Ther 2012 Jun; 10(6): 701-706. doi: 10.1586/eri.12.50.

3. Angus DC, Wax RS. Epidemiology of sepsis: an update. Crit Care Med 2001; 29 (7 suppl): S109-S116. 
4. Becker JU, Theodosis C, Jacob ST, Wira CR, Groce NE. Surviving sepsis in low-income and middle-income countries: new directions for care and research. Lancet Infect Dis 2009 Sep; 9(9): 577-82.

5. Cheng AC, West TW, Limmathurotsakul D, Peacock SJ. Strategies to reduce mortality from bacterial sepsis in adults in developing countries. PLoS Med 2008 Aug; 5(8): e175. doi: 10.1371/journal. pmed.0050175.

6. Silva E, Pedro Mde A, Sogayar AC, Mohovic T, Silva CL, Janiszewski M, et al. Brazilian Sepsis Epidemiological Study (BASES study). Crit Care Aug; 8(4): R251-60.

7. Bane A, Yohannes AG, Fekade D. Morbidly and mortality of adult patients with HIV/AIDS at Tikur Anbessa Teaching Hospital, Addis Ababa, Ethiopia. Ethiop Med J 2003 Apr; 41(2): 131-40.

8. Sani MU, Mohammed AZ, Adamu B, Yusuf SM, Samalia AA, Borodo MM AIDS mortality in a tertiary health institution: a Four-Year Review. J Natl Med Assoc 2006 Jun; 98(6): 862-6.

9. Bozza FA, Salluh JI. An urban perspective on sepsis in developing countries. Lancet Infect Dis 2010 May; 10(5): 290-1. doi: 10.1016/ S1473-3099(10)70074-8.

10. Silva E, Dalfior Junior L, Fernandes HD, Moreno R, Vincent JL. Prevalence and outcomes of infections in Brazilian ICUs: a subanalysis of EPIC II study. Revista Brasileira de Terapia Intensiva 2012 Jun; 24:143-150
11. Conde KA, Silva E, Silva CO, Ferreira E, Freitas FG, Castro I, et al. Differences in sepsis treatment and outcomes between public and private hospitals in Brazil: a multicenter observational study. PLoS ONE 2013 Jun; 8:e64790. doi: 10.1371/journal.pone.0064790.

12. Li ZQ, Xi XM, Luo X, Li J, Jiang L. Implementing Surviving Sepsis Campaign bundles in China: a prospective cohort study. Chin Med J (Engl). 2013; 126:1819-1825.

13. Rhodes A, Bennet ED. Early goal-directed therapy: an evidence-based review. Crit Care Med 2004; 32: S448.

14.Weippi G. Infectioius toxic hypotension-effect and dosage of midodrine. Padiatr Pado. 1979; 14(2): 211-6.

15. Reddy EA, Shaw AV, Crump JA. Community-acquired bloodstream infections in Africa: a systemic review and meta-analysis. Lancet Infect Dis 2010 Jun; 10(6): 417-32. doi: 10.1016/S1473-3099(10)70072-4.

16. Noritomi DT, Ranzani OT, Monteiro MB, Ferreira EM, Santos SR, Leibel $\mathrm{F}$, et al. Implementation of a multifaceted sepsis education program in an emerging country setting: clinical outcomes and cost effectiveness in a long-term follow-up study. Intensive Care Med. 2014; 40:182191. doi: 10.1007/s00134-013-3131-5.

17. Baelani I, Jochberger S, Laimer T, Otieno D, Kabutu J, Wilson I, et al. Availability of critical care resources to treat patients with severe sepsis or septic shock in Africa: a self-reported, continent-wide survey of anaesthesia providers. Crit Care. 2011; 15:R10. doi: 10.1186/ cc9410. 